\title{
Determinants of measured olfactory sensitivity
}

\author{
MICHAEL D. RABIN and WILLIAM S. CAIN \\ Yale University and John B. Pierce Foundation Laboratory, New Haven, Connecticut
}

\begin{abstract}
Ten participants sought to detect four odorants: benzaldehyde, pyridine, and two alcohols, $n$-butyl and n-amyl alcohol, that smelled similar to each other. These were presented repeatedly on 3 successive days. The sequence of testing during a session made it possible to determine whether experience with one odorant would specifically facilitate the detectability of a similar-smelling odorant and whether any such facilitation would restrict itself to the nostril through which the experience was gained. Neither of these possibilities occurred. Instead, measured sensitivity increased rather uniformly both within and across days. Net gain from beginning to end exceeded an order of magnitude. Averaging across sessions gave a picture of smaller than usual individual differences, under 20 to 1 , attributable mainly to general rather than odorant-specific differences in sensitivity. The results indicate that thresholds gathered in customary brief testing will underestimate olfactory sensitivity and overestimate individual differences. Incorporation of a reference odorant into threshold experiments should increase comparability among studies.
\end{abstract}

Olfactory sensitivity as typically measured in the laboratory varies markedly from one person to another (Punter, 1983). Based on his own extensive data, Amoore (1980) developed the rule of thumb that about $96 \%$ of the population falls between $1 / 16$ and 16 times the mean threshold for an odorant, that is, within a 256-fold range.

Does such high variation reflect only individual differences in absolute sensitivity? Substantial extraneous variation could occur from poor stimulus control, unsophisticated psychophysical methodology, or collection of insufficient data. Studies of olfactory threshold have often relied, for instance, on a single brief determination of threshold per participant. Even with more thorough measurements, however, reliability has received scant attention. Hence, we know little about whether variability between people commonly exceeds variability within a person tested at different times.

Observers may differ in sensitivity in two ways: (1) One observer may prove more sensitive than another to virtually all odorants. This situation could reflect the operation of some general factor, including something as simple as how many molecules can gain access to the receptors within the nasal cavity. (2) An observer may prove more sensitive than average to some odors and less sensitive to others. If observers show idiosyncratic yet stable disparities, then the possible explanations could prove numerous.

The present study addressed the issue of individual variation and reliability, using standard threshold methodology to gather observations on four odorants for the same individuals both within a day and across days. The testing thereby allowed a glimpse of whether participants

This study was supported by Grants ES 00592 and NS 21644 from the National Institutes of Health. We thank Ruth Isseroff for assistance. Address correspondence to: M. D. Rabin, John B. Pierce Foundation Laboratory, 290 Congress Avenue, New Haven, CT 06519. would show systematic changes both over time and across odorants. The sequence of testing within a day was designed to permit conclusions about the possible mechanism and specificity of any gains in performance.

\section{METHOD}

\section{Participants}

Five nonsmoking males and 5 nonsmoking females between the ages of 22 and 28 participated. None had previously served in experiments on absolute sensitivity for smell and none had any history of olfactory dysfunction.

\section{Materials}

The odorants (reagent grade) were n-butyl alcohol (BA), Mallinckrodt; n-amyl alcohol (AA), Mallinckrodt; benzaldehyde (BZ), Kodak; and Pyridine (PY), Matheson. All had nominal purity of at least $99 \%$. n-butyl alcohol and n-amyl alcohol were selected because of their similarity to one another and their dissimilarity to the other two odorants.

Stimulus delivery occurred via squeezable polyethylene bottles (250 ml) (Cain, Gent, Catalanotto, \& Goodspeed, 1983). Their closures had flip-up spouts that could deliver odorized air into either nostril singly or, when the bottle was waved beneath the nose, into both nostrils simultaneously.

A nine-bottle dilution series ( $60 \mathrm{ml}$ per bottle) was prepared for each odorant, using deionized water as diluent. Dilutions progressed in ternary steps from $4.0 \%$ to $0.00020 \%(v / v)$ for n-butyl alcohol, $2.0 \%$ to $0.00010 \%$ for n-amyl alcohol, $0.1 \%$ to $0.0000052 \%$ for benzaldehyde, and $0.2 \%$ to $0.000010 \%$ for pyridine. The highest concentration in each series roughly matched the perceived intensity of $4.0 \%$ n-butyl alcohol. In addition to the bottles that contained odorant, 16 bottles of deionized water served as blanks.

Dilution series were prepared daily in duplicate so that no single bottle in a series would be presented to an observer twice successively. Chromatographic measurements implied that headspace concentration reestablished itself within $1 \mathrm{~min}$ after a puff of odorant.

\section{Procedure}

Threshold testing followed a forced-choice procedure. The participant received three bottles in random order: one contained a solution of odorant and the other two just water. The participant had to select the bottle with odorant and received neither familiariza- 


$\begin{array}{ccccccccccc}1 & 2 & 3 & 4 & 5 & 6 & 7 & 8 & 9 & 10 & - \text { Bbok\# } \\ \text { AA } & \text { BZ } & \text { BA } & \text { PY } & \text { BA } & \text { PY } & \text { BA } & \text { PY } & \text { AA } & \text { BZ } & \text { - Odorant }\end{array}$

LRLR LRLR LLLL BBBB LLLL BBBB LLLL BBBB RLRL RLRL - Nostril

$12345678 \ldots$

.....40- Senies \#

Figure 1. Sequence of a test session. $L=$ left nostril; $R=$ right nostril; $B$ = both.

tion nor feedback. On a trial, the participant picked up the first bottle, opened the cap, held it approximately $1 \mathrm{~cm}$ below the designated nostril, and squeezed the bottle while sniffing. The experimenter promoted uniform inhalations by visual monitoring and encouragement. During presentation to a single nostril, the participant held the contralateral nostril shut. During presentation to both nostrils, the participant gently waved the bottle beneath both nostrils while sniffing. After sampling the three bottles in this way, the participant chose one. Bottles could be sampled only once per trial.

Testing proceeded according to an ascending sequence of concentrations in order to minimize adaptation. A series began with the lowest concentration. If the participant chose incorrectly, the next higher concentration was presented. If the participant chose correctly, the same concentration was repeated. Three correct choices at the same concentration marked a threshold, halted testing on that series, and initiated testing on the next series.

Sessions lasted approximately $3 \mathrm{~h}$ on each of 3 successive days. A session comprised 40 threshold determinations in 10 blocks of four determinations per odorant. Participants had the chance to rest between blocks. The sequence of a session is shown in Figure 1.
Half the time the left nostril was used first, and half the time the right nostril was used first; for Blocks 3,5, and 7, half the participants used the left nostril and the other half the right nostril.

The sequence of testing allowed us to inspect the following: (1) whether progressive experience throughout a series would lead to gains in detection in comparisons of $\mathrm{AA}(1)$ versus $\mathrm{AA}(9), \mathrm{BZ}(2)$ versus $\mathrm{BZ}(10), \mathrm{BA}(3)$ versus $\mathrm{BA}(5)$ versus $\mathrm{BA}(7)$, and $\mathrm{PY}(4)$ versus PY(6) versus PY(8); (2) whether experience with a similar-smelling odorant would facilitate detection of a test odorant, and hence, whether the gain, if any, between $\mathrm{AA}(1)$ and $\mathrm{AA}(9)$ would exceed that between $B Z(2)$ and $B Z(10)$ because of the interpolated experience with the alcohol in $\mathrm{BA}(3)-\mathrm{BA}(5)-\mathrm{BA}(7)$; and (3) whether any gain between $\mathrm{AA}(1)$ and $\mathrm{AA}(9)$ would restrict itself to the nostril that had received the interpolated experience to BA in Blocks 3,5 , and 7 .

\section{RESULTS}

Figure 2 depicts how threshold varied, block by block, throughout the 3 days of testing. Benzaldehyde produced the lowest thresholds and the two alcohols the highest. For any given odorant, threshold tended to decline both within a day and from day to day. That is, experience increased apparent sensitivity. The net gain in sensitivity from the first threshold for an odorant in the first session to the last threshold for that odorant in the third session averaged a factor of $13(S D=8.3)$.

The thresholds shown for amyl alcohol and benzaldehyde in Figure 2 represent averages taken across single

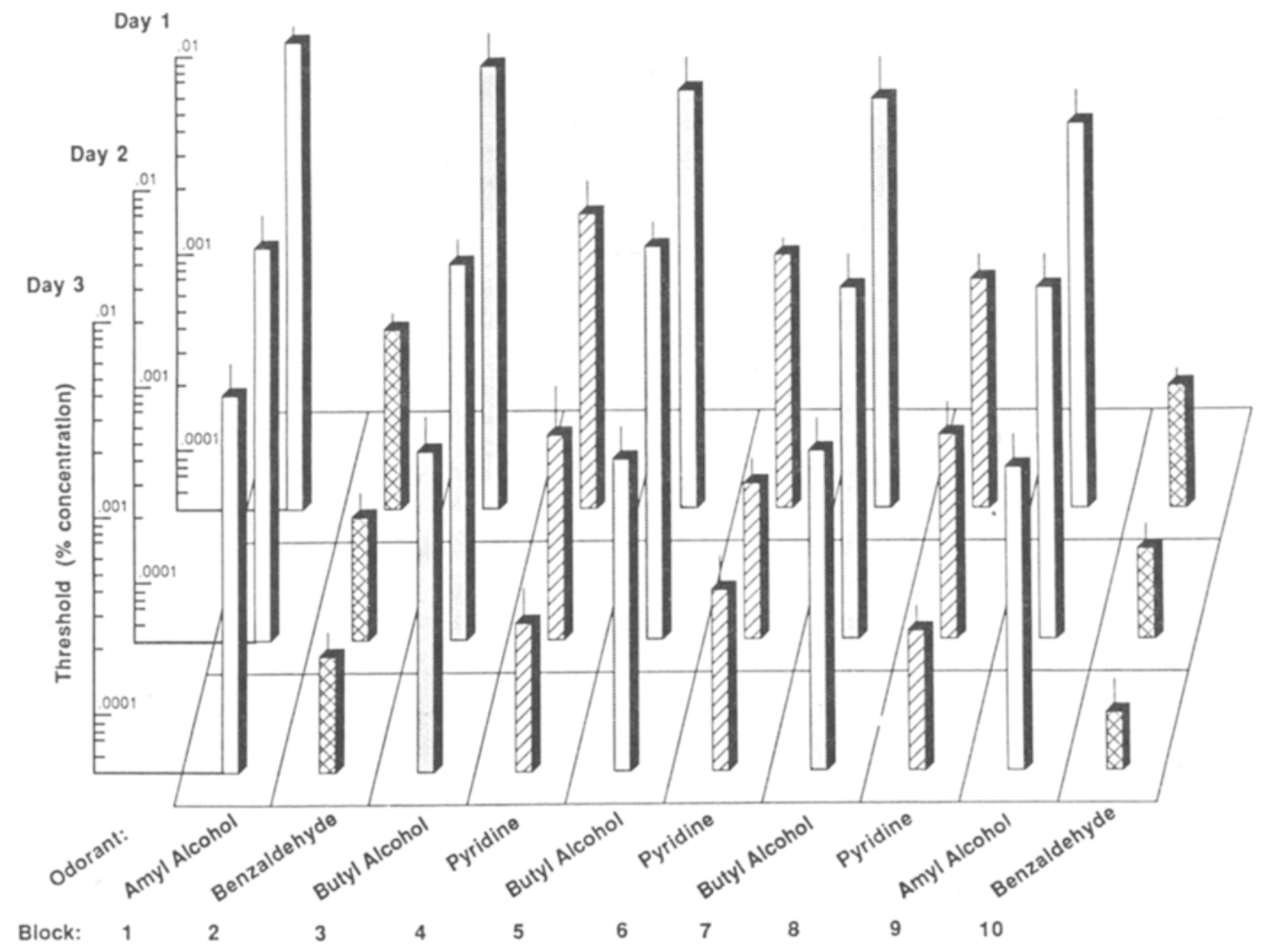

Figure 2. Thresholds (+1 SEM) measured over blocks and days. 
nostrils without respect to which had received interpolated exposure to butyl alcohol. Nostril-specific exposure to butyl alcohol had yielded neither nostril-specific sensitization nor desensitization to either its homologue, amyl alcohol, or the unrelated compound benzaldehyde (Figure 3). Each nostril and each odorant changed over time to the same degree.

Although threshold tended to decrease across blocks for all four odorants, only the reduction for amyl alcohol and benzaldehyde achieved significance $[F(3,27)=9.59$, $p<.001$ for amyl alcohol and benzaldehyde; $F(6,54)=$ $1.43, p=.22$ for butyl alcohol and pyridine]. Such differences, however, hardly imply odorant-specific effects of experience. They reflect chiefly the size of the interval between successive tests (Figure 4). Eight blocks separated successive tests of amyl alcohol and benzaldehyde, whereas two blocks separated successive tests of butyl alcohol and pyridine. To a first approximation, it appears that simple experience, irrespective of odor type, determines gain in apparent sensitivity.

Reduction of average thresholds day by day followed much the same time-course for three of the four odorants, and a somewhat slower course for benzaldehyde (Figure 5). This seeming difference did not, however, show up as a significant interaction in an ANOVA $[F(3,27)=89.0, p<.001$ for odorant; $F(2,18)=14.0$, $p<.001$ for day; and $F(6,54)=0.91, p=.3$ for odorant-by-day interaction]. Irrespective of odorant, it appeared that threshold would have continued to fall with further testing. Throughout the reduction from Day 1 through Day 3, the standard error of the measurements remained approximately the same, that is, the participants

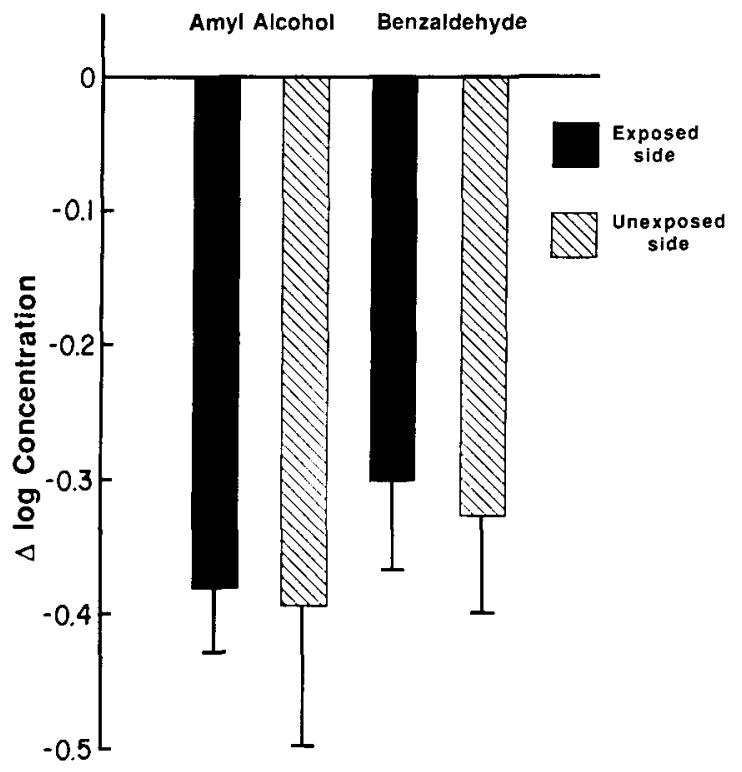

Figure 3. Change in thresholds for n-amyl alcohol and benzaldehyde in nostrils that did or did not receive interpolated exposure to n-butyl alcohol.

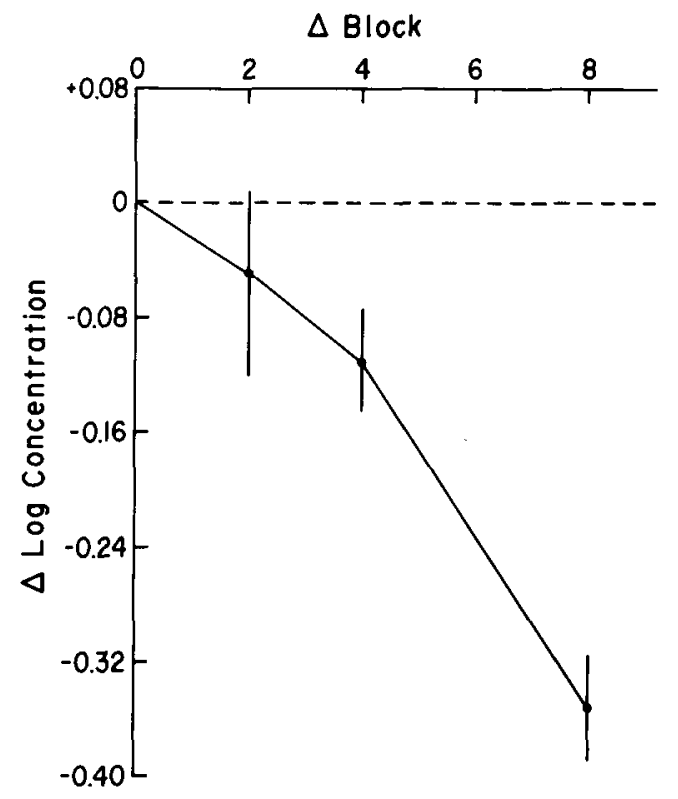

Figure 4. Change in threshold versus the number of blocks separating tests. Line depicts average changes across odorants; bars depict range.

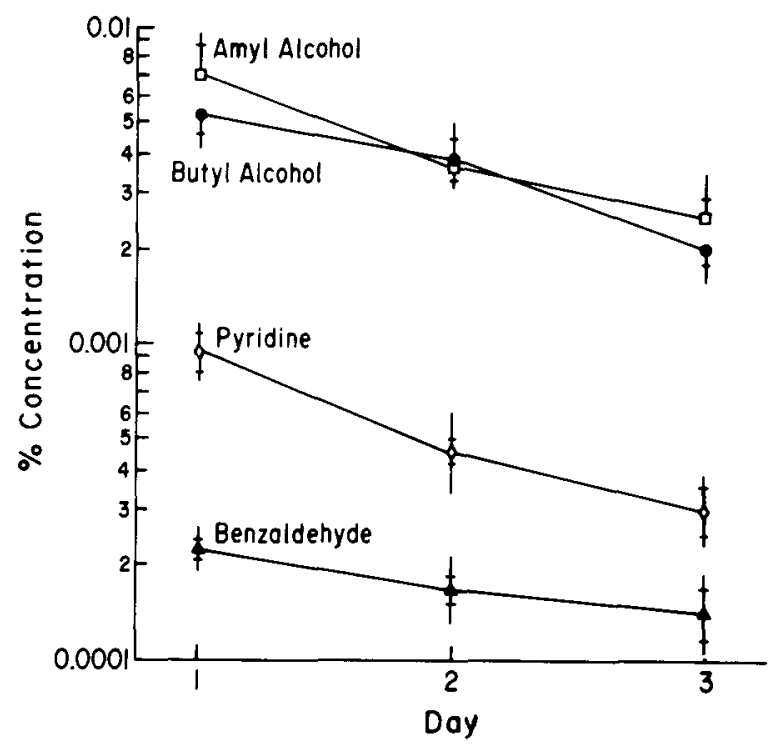

Figure 5. Thresholds (averages across blocks) versus days. Bars depict SEM for non-normalized data (full length) and normalized data (indicated segment).

exhibited no tendency to perform more similarly to one another.

Table 1 shows the range and variance of the average threshold for each odorant across participants. The values exhibited relative uniformity from one odorant to another. In order to decide whether these measures of dispersion represented general or specific differences in sensitivity, the data were normalized to the grand geometric mean 
Table 1

Variability of Thresholds Before and After Normalization

\begin{tabular}{lccccc}
\hline \multicolumn{1}{c}{ Odorant } & $\begin{array}{c}\text { Threshold } \\
(\log \%)\end{array}$ & $\begin{array}{c}\text { Variance } \\
(\log )\end{array}$ & $\begin{array}{c}\text { Range } \\
\text { (linear) }\end{array}$ & $\begin{array}{c}\text { Normalized } \\
\text { Variance } \\
(\log )\end{array}$ & $\begin{array}{c}\text { Normalized } \\
\text { Range } \\
\text { (linear) }\end{array}$ \\
\hline Amyl alcohol & -2.343 & 0.358 & 18.5 & 0.083 & 3.6 \\
Butyl alcohol & -2.440 & 0.372 & 14.6 & 0.053 & 3.2 \\
Benzaldehyde & -3.705 & 0.195 & 6.1 & 0.142 & 6.5 \\
Pyridine & -3.292 & 0.255 & 12.0 & 0.081 & 3.6 \\
\hline
\end{tabular}

across participants and odorants. This operation entailed multiplying each participant's thresholds by whatever factor brought the geometric mean of his or her thresholds across odorants into register with that of the entire group. Normalized ranges and variances appear on the right side of Table 1 . These entries fall considerably below those on the left, except in the case of benzaldehyde.

The difference between the normalized and nonnormalized variance in Table 1 allowed an estimate of the percentage variance contributed by general differences in sensitivity. This equaled $77 \%$ for amyl alcohol, $86 \%$ for butyl alcohol, $27 \%$ for benzaldehyde, and $68 \%$ for pyridine. Figure 6 displays the normalized thresholds for the 10 participants. The residual variability shown here reflects all sources other than general sensitivity (e.g., stimulus noise, error of psychophysical measurement, and odorant-specific differences in sensitivity).

There is more than one way to compute the relative stability of performance from one occasion to another. One way entails computation of the correlation coefficient across the four odorants from session to session for each

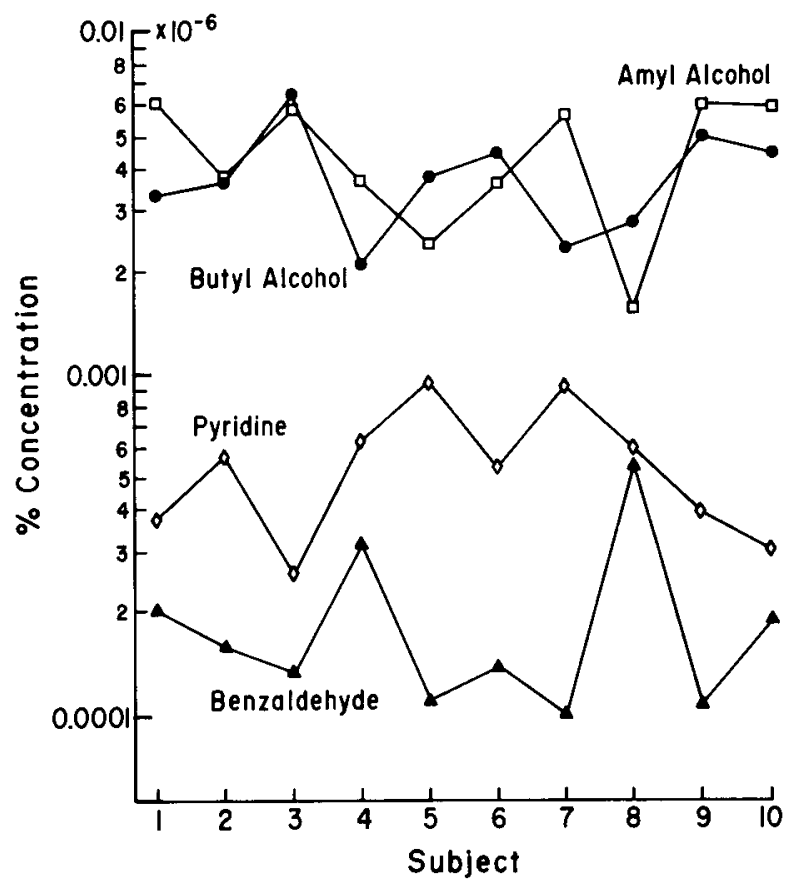

Figure 6. Normalized thresholds for the 10 participants plotted separately. participant. The distribution of such coefficients yielded medians of $0.97(Q=0.05)$ for Days 1 to $2,0.95(Q$ $=0.12)$ for Days 1 to 3 , and $0.96(Q=0.03)$ for Days 2 to 3 . This outcome implies small session-to-session variation in comparison with the average differences in threshold from odorant to odorant.

When looked at one odorant at a time across participants, the distribution yielded the more modest median value of $0.61(Q=0.08)$. This outcome implies large session-to-session variability per participant in comparison to the range of sensitivity across participants. In the present case, where the range across participants was rather small, however, random variation from session to session had only a modest effect on absolute precision. A test of session-to-session variation would be more meaningful if performed on participants with a wider range of sensitivity, such as clinical patients.

\section{DISCUSSION}

High person-to-person variability in olfactory sensitivity may be largely an illusion. The impression of high variability probably arises from the collection of insufficient data per person in most experiments. The temptation to collect insufficient data stems from the large investment of time necessary to achieve stability in the data. Experimenters interested in reasonably exact specification of a threshold have characteristically studied relatively large groups of participants, often over 100 , very briefly. In such studies, threshold usually varies over a range of a few hundred or even a thousand to one (Amoore, 1977; Amoore, Forrester, \& Buttery, 1975; Amoore, Pelosi, \& Forrester, 1977; Jones, 1957). The present range, under 20 to 1 , therefore falls well below most published values. The small size and uniformity of age of the sample warrants caution regarding any estimate of the true range. Nevertheless, some matters are not in doubt. One is that the range will decrease, as it did here, with the collection of more data. In any individual block, the range sometimes exceeded 100 to 1 , but averaging across blocks or days reduced the range to the comparatively low values shown in Table 1.

Although averaging larger amounts of data per participant reduces the estimate of person-to-person variability, the collection of such data over time reveals how thresholds may change systematically. Repeated testing in olfaction results in more than just reduction of random error; it results in different thresholds. A large sample of participants studied only superficially cannot, therefore, substitute for a sample of whatever size studied more thoroughly. In cases where relative threshold values alone are of interest, a large sample briefly studied may suffice. In those many other cases where the absolute value holds interest, brief study may overestimate the threshold by more than an order of magnitude.

Absolute, rather than relative, values often hold particular interest in applied settings, such as the industrial workplace, where detection of an odor through a respi- 
rator may signal danger; in dispersion modeling of odor pollution, where the threshold may determine means of abatement; or in flavor analysis. The particular threshold value relevant in a real-world setting will, of course, depend on many factors, including the sophistication and preparedness of the recipient. Threshold values that appear in the odor literature are often compiled into tables for such applications (Fazzalari, 1978; van Gemert \& Nettenbreier, 1977). Users of the tables commonly complain that the study-to-study variability is so high that the values become more a source of frustration than a help (see Amoore \& Hautala, 1983).

The results of studies by Punter (1983) and Engen (1960) anticipated some of those in the present study. Punter found high test-retest correlations for individual participants across odorants (mean $=0.95$ ), but lower correlations for individual odorants (median $=0.40$ ). Comparable values here equaled 0.97 and 0.61 , respectively. Hence, in the normal course of threshold measurement, differences in potency between odorants will tend to predominate over any unreliability in measurements taken at any one time, although the unreliability should be reckoned with by sufficient testing. Finally, neither we nor Punter found significant differences between males and females $[F(1,8)=3.88, p>.05$ for sex].

In Engen's (1960) study, in which 5 participants sought to detect six odorants presented sequentially from one 3 day period to another (amyl acetate for 3 days followed by phenylethyl alcohol for 3 days followed by heptane for 3 days, etc.), threshold for each successive odorant decreased systematically during testing up to the fifth. Engen concluded that practice with one odorant transferred to the next. The data suggest at least partial, if not complete, transfer, and thereby imply that experience with the same odorant would yield some gain in apparent sensitivity beyond 3 days. The present data support both the notion of transfer and the notion of continued gain beyond 3 days despite extensive experience on those days. Indeed, the continued gain in apparent sensitivity throughout the 3 days thwarted our hope to obtain estimates of the dispersion within individuals tested repeatedly.

Engen (1960) suggested that improvement in apparent sensitivity may have arisen from participants' realization that they need not recognize odor quality in order to detect correctly. Indeed, he then demonstrated that apparent sensitivity would improve drastically when a participant went from a "recognition" decision to a "detection" decision, even in a forced-choice situation. Semb (1968), who found a 20-fold gain in detection for 2 participants over 500 trials on an unspecified number of days, offered no speculations regarding the gain. His use of a double random staircase and partial feedback makes it difficult to know whether the recognitiondetection dichotomy had any relevance. Nevertheless, it seems unlikely that this narrow dichotomy will explain all instances of gain.
If the present investigation makes any contribution to the search for a mechanism, it lies in the demonstration that the gain lacks a peripheral origin, since it transfers from a stimulated to an unstimulated nostril and transfers readily from one odor quality to another (Figure 3). Whether the gain will prove explainable entirely by changes in response criterion, or whether the explanation will need to invoke more general processes involved in perceptual learning, remains to be seen. Experiments showing how apparent sensitivity develops over time with feedback and payoff could elucidate the contribution of response criterion (Engen, 1972).

In summary, this investigation calls into question the common notion of large individual differences in olfactory sensitivity. Although there seem to be true differences of a general nature, their size diminishes as the amount of data collected per participant increases. This is merely a statistical matter, rather than a true convergence of thresholds over time. Repeated testing causes a steady downward drift in measured threshold over days. The drift implies that brief measurements will overestimate threshold as well as exaggerate apparent individual differences. On a practical level, it will often prove impossible to measure sensitivity over a long enough period to ensure that participants have achieved asymptotic performance. Accordingly, the use of a reference odorant could prove helpful for subsequent comparability. Measurement of the threshold to the reference odorant should follow the same protocol as measurement of the threshold to test odorants. n-butyl alcohol commonly serves as a reference in suprathreshold measurements and could undoubtedly serve well in threshold measurements also (Moskowitz, Dravnieks, Cain, \& Turk, 1974).

\section{REFERENCES}

Amoore, J. E. (1977). Specific anosmia and the concept of primary odors. Chemical Senses \& Flavor, 2, 267-281.

Amoore, J. E. (1980). Properties of olfactory system. In F. H. Suchomel \& J. W. Weatherly III (Eds.), Odorization (pp. 31-35). Chicago: Institute of Gas Technology.

Amoore, J. E., Forrester, L. J., \& Buttery, R. G. (1975). Specific anosmia to 1-pyrroline: The spermous primary odor. Joumal of Chemical Ecology, 1, 299-310.

Amoore, J. E., \& Hautala, E. (1983). Odor as an aid to chemical safety: Odor thresholds compared with threshold limit values and volatiles for 214 industrial chemicals in air and water dilution. Journal of Applied Toxicology, 3, 272-289.

Amoore, J. E., Pelosi, P., Forrester, L. J. (1977). Specific anosmia to 5 a-androst-16-en-3-one and o-pentadecalactone: The urinous and musky primary odors. Chemical Senses \& Flavor, 2, 401-425.

Cain, W. S., Gent, J., Catalanotto, F. A., \& Goodspeed, R. B. (1983). Clinical evaluation of olfaction. American Joumal of Otolaryngology, 4, 252-256.

ENGEN, T. (1960). Effect of practice and instruction on olfactory thresholds. Perceptual \& Motor Skills, 10, 195-198.

ENGEN, T. (1972). The effect of expectation on judgments of odor (Research Report ICRL-RR-70-11). Washington, DC: U.S. Department of Health, Education and Welfare, Injury Control Research Laboratory.

FAzZALARI, F. A. (1978). Compilation of odor and taste threshold values data. Philadelphia: American Society for Testing and Materials. 
JoNEs, F. N. (1957). An analysis of individual differences in olfactory thresholds. American Journal of Psychology, 70, 227-232.

Moskowitz, H. R., Dravnieks, A., Cain, W. S., \& TURK, A. (1974). A standardized procedure for expressing odor intensity. Chemical Senses \& Flavor, 1, 235-237.

PunTer, P. H. (1983). Measurement of human olfactory thresholds for several groups of structurally related compounds. Chemical Senses, 7, 215-235.
SEmB, G. (1968). The detectability of the odor of butanol. Perception \& Psychophysics, 4, 335-340.

VAN Gemert, L. J., \& NetTenbreier, A. H. (1977). Compilation of odour threshold values in air and water. Zeist, The Netherlands: CIVO.

(Manuscript received February 15, 1986; revision accepted for publication March 30, 1986.)

\title{
Announcements
}

\author{
16th Annual Meeting \\ Society for Computers in Psychology
}

The 16th Annual Meeting of the Society for Computers in Psychology will be held at the Hyatt Regency Hotel, New Orleans, Louisiana, November 12, 1986. The meeting features papers on applications of computers to all areas of psychology-experimental, clinical, and educationalwith the emphasis on on-line, laboratory applications. Contact: Cyndi McDaniel, Psychology Department, Northern Kentucky University, Highland Heights, Kentucky 41076; (606) 572-5310.

\section{Call for Papers \\ Society for Computers in Psychology}

Papers and symposia are sought for the 16th Annual Meeting of the Society for Computers in Psychology, November 12, 1986, New Orleans, Louisiana. Deadline for papers: July 1, 1986. For more information, contact Cyndi McDaniel, Psychology Department, Northern Kentucky University, Highland Heights, Kentucky 41076; (606) 572-5310.

\section{Student Paper Competition Society for Computers in Psychology}

The Society for Computers in Psychology will continue to sponsor an award for the outstanding student paper submitted for presentation at the annual meeting. Although the primary emphasis of the meeting is the use of computers in on-line, experimental applications, student papers in any area of the application of computers to psychology are welcome. Papers may be theoretical, experimental, or applied in approach. Eligibility is open to (1) work done by a student currently enrolled in undergraduate or graduate courses or (2) work done as part of a course, thesis, or other student research by a person who has graduated in 1986. All papers submitted for the meeting (including multiply authored ones) in which the major contribution has been made by a student are eligible for the prize, and will be considered for presentation at the meeting and subsequent publication. The winning paper will be presented at the 1986 meeting, and the author will receive a complimentary 1-year membership in the meeting, a complimentary 1-year subscription to $\mathrm{Be}$ havior Research Methods, Instruments, \& Computers, and a \$50 cash prize. Deadline for papers: July $1,1986$.

Eligible papers should be submitted in quadruplicate. A cover sheet should include the author's name, mailing address, telephone number, and academic affiliation, a 50-word abstract, and a note stating that the paper is to be considered for the student award. These materials should be sent to: Cyndi McDaniel, Psychology Department, Northern Kentucky University, Highland Heights, Kentucky 41076; (606) 572-5310.

The winner of the 1985 competition was Brian Watts of New York University, whose paper is published in these Proceedings. 